\title{
Comparison of The Effectiveness of Probiotic and Chlorhexidine Mouth Rinses on Plaque Accumulation and Gingival Inflammation
}

\author{
Kumar Vivek, Amrita, Pratap Mahender \\ ${ }_{1}^{1}$ Department of Periodontology, Hazaribag College of Dental Sciences and Hospital, Demotand, \\ Hazaribag, Jharkhand, India. E-mail: drvivek1909@gmail.com \\ 2 Department of Periodontology, Uttaranchal Dental and Medical research Institute, Majri Grant, \\ Doiwala, Dehradun, Uttarakhand, India. E-mail: dramritaperio@gmail.com \\ 3Department of Orthodontics and Dentofacial Orthopaedics, Uttaranchal Dental and Medical \\ research Institute, Majri Grant, Doiwala, Dehradun, Uttarakhand, India. \\ E-mail: samratpratapi@gmail.com
}

\section{ABSTRACT}

Background: Chemical agents have been increasingly used as an adjunct to mechanical control. The use of a mouthwash augments maintenance of oral health through its antiplaque and antibacterial chemical properties The aim of the study was to compare the effectiveness of probiotic and chlorhexidine mouth rinses on plaque accumulation and gingival inflammation.

Methods: A total of 60 patients with chronic gingivitis were divided into 3 groups. Group A - 20 subjects were advised probiotic mouthwash, Group B - 20 subjects were advised chlorhexidine mouthwash and Group C - 20 subjects into a negative control group (normal saline). Oral prophylaxis was done for all groups at baseline. Baseline scores of plaque index (PI) gingival index (GI) and oral hygiene indexsimplified (OHI-S) were recorded. The designated mouth rinses were distributed to the respective groups and they were instructed to rinse twice daily. Variables were again recorded on the seventh and 14th day after use of mouthwashes and data obtained was subjected to statistical analysis.

Results: A significant difference in PI, GI, and OHI(S) were found when group 1 and group II was compared with group 3 on day14 and day28. However, there were no significant differences in the mean $\mathrm{PI}, \mathrm{GI}$ and $\mathrm{OHI}(\mathrm{S})$ between the Probiotic and Chlorhexidine groups on the 14th day of examination.

Conclusion: The Probiotic mouth rinse was found to be effective in reducing plaque accumulation and gingival inflammation. Therefore, the Probiotic mouth rinse has a potential therapeutic value and further long-term study is recommended to determine its efficacy.

Keywords: chlorhexidine, probiotics, gingivitis, plaque

\section{Introduction}

The most prevalent infectious oral diseases in humans, periodontal diseases, are associated with dental plaque. The level and type of bacteria in the oral cavity predispose to the recurrence of infection. Long term antibiotic use has been considered but has resulted in resistance and other undesirable side effects. Antiplaque agents function by removing or disrupting biofilms, or by preventing the formation of new biofilm. Using mouthwashes is an effective and safe method for delivery of antimicrobial agents and they are 
being used widely. These agents are capable of preventing bacterial adhesion, colonization, and metabolism, and thus affect bacterial growth.[1]

Probiotics may be a promising area of research in periodontal therapy. WHO[2] describes probiotics as "live microorganisms which, when administered in adequate amounts in food or as dietary supplement confer a health benefit on the host". This term has been derived from the Greek language which means "for life".[3] The term Probiotic, as an antonym to the term antibiotic, was first used by Lilly and Stillwell to describe substances secreted by one microorganism which stimulates the growth of another. They repopulate the beneficial bacteria, which can help kill pathogenic bacteria and fight against infection. ${ }^{[4]}$

Patient motivation is of utmost importance in mechanical plaque control. Chemical plaque control agents act as adjuncts to conventional mechanical plaque control and interfere with biofilm composition and metabolism. Chlorhexidine (CHX) is one of the most effective antimicrobial agents for plaque control.[5,6] Chlorhexidine is regarded as a gold standard in dentistry for the prevention of dental plaque. It has certain side effects such as brown discoloration of teeth, oral mucosal erosion, and bitter taste.[7]

To overcome the drawbacks of antimicrobial chemical agents, probiotic therapy can be considered as a viable alternative in the prevention of oral disease. In light of the above facts, the current study was designed with an aim to compare the effectiveness of probiotic and chlorhexidine mouth rinses on plaque accumulation and gingival inflammation.

\section{Materials and Methods}

A randomized, parallel group clinical study was performed from March 2017 to September 2017 in the Department of Periodontology after obtaining ethical approval from the institutional review board of the college. A total of 60 male and female subjects aged between 25 and 35 years participated in this study. Written informed consent was taken from all the participants before the start of the study.

\section{Inclusion criteria}

- Subjects should have at least 24 evaluable teeth

- Subjects with chronic gingivitis

\section{$\underline{\text { Exclusion criteria }}$}

(1) cigarette smoking or tobacco use and alcoholism;

(2) systemic diseases such as diabetes mellitus, hypertension, and rheumatoid arthritis;

(3) pregnancy;

(4) systemic bacterial, viral, or fungal infection;

(5) history of antibiotic therapy or use of anti-inflammatory medications during the past 6 months;

(6) periodontal therapy during the past 2 years;

(7) Subjects with mouth breathing habit; and

(8) Subjects with orthodontic and prosthodontic appliances

Baseline plaque and gingival inflammation score were calculated using the plaque index (Silness and Loe), gingival index (Loe and Silness) and oral hygiene index-simplified (Green and Vermillion) by a single examiner. Thorough oral prophylaxis was performed and all participants were taught to use a modified bass technique for brushing. The patients were randomly divided into three groups consisting of 15 patients each as under:

Group A - Probiotic mouthrinse

Group B - Chlorhexidine mouthwash $0.2 \%$

Group C - Control (saline) 
The designated mouthwashes were distributed to the respective groups; Darolac sachets (Lactobacillus acidophilus, Lactobacillus rhamnosus, Lactobacillus sporogenes, Bifidobacterium longum, and Saccharomyces boulardii ) were diluted in $10 \mathrm{~mL}$ of distilled water and used as a mouthwash. $10 \mathrm{~mL}$ of chlorhexidine mouthwash (Rexidin, Warren-Indoco Remedies Ltd, Mumbai, India) without any dilution rinsed for 1 minute. All the three groups were advised to rinse their mouths with the respective mouthwashes prescribed to them for 14 days without any dilution for 1 min twice daily half an hour after brushing. The subjects were asked not to eat or drink anything for the next half an hour to achieve the effect of the mouthwash. All the subjects were asked to report to the department on 14th and 28th day for a recording of the variables.

\section{Data Analysis}

Statistical analyses were performed using SPSS software version 21 (SPSS Inc., Chicago, IL, USA). Intergroup comparison was done using the Mann-Whitney test and t-test. $\mathrm{P}<0.05$ was considered statistically significant. Data were presented as mean \pm standard deviation.

\section{Results}

The mean PI values for all three mouth rinses were 0 at baseline as scaling and polishing were done for all tooth surfaces. The mean values of PI score after14 days is $0.08 \pm 0.03$ in the probiotic group, $0.06 \pm 0.03$ in chlorhexidine group and $0.85 \pm 0.27$ in control group and after 28 days the mean value of PI is $0.42 \pm 0.21$ in the probiotic group, $0.25 \pm 0.15$ in chlorhexidine group and $1.16 \pm 0.32$ in control group. The mean values of GI score after14 days is $0.07 \pm 0.04$ in the probiotic group, $0.06 \pm 0.03$ in chlorhexidine group and $0.97 \pm 0.37$ in control group and after 30 days the mean value of PI is $0.45 \pm 0.18$ in the probiotic group, $0.29 \pm 0.11$ in chlorhexidine group and $1.03 \pm 0.18$ in control group. The mean values of $\mathrm{OHI}(\mathrm{S})$ score after14 days is $0.25 \pm 0.09$ in the probiotic group, $0.20 \pm 0.12$ in chlorhexidine group and $0.73 \pm 0.26$ in control group and after 28 days the mean value of PI is $0.48 \pm 0.28$ in the probiotic group, $0.32 \pm 0.18$ in chlorhexidine group and $1.17 \pm 0.37$ in control group as shown in table 1 .

\begin{tabular}{|l|l|l|l|l|}
\hline \multicolumn{5}{|c|}{ Table 1. Values (mean \pm SD) of PI, GI, OHI(S) in the three Groups } \\
\hline \multirow{2}{*}{ Variable } & Interval & Group 1 & Group 2 & Group 3 \\
\hline \multirow{3}{*}{ PI } & Baseline & 0 & 0 & 0 \\
& Day14 & $0.08 \pm 0.03$ & $0.06 \pm 0.03$ & $0.85 \pm 0.27$ \\
& Day28 & $0.42 \pm 0.21$ & $0.25 \pm 0.15$ & $1.16 \pm 0.32$ \\
\hline \multirow{3}{*}{ GI } & Baseline & $0.12 \pm 0.08$ & $0.18 \pm 0.09$ & $0.15 \pm 0.06$ \\
& Day14 & $0.07 \pm 0.04$ & $0.06 \pm 0.03$ & $0.97 \pm 0.37$ \\
& Day28 & $0.45 \pm 0.18$ & $0.29 \pm 0.11$ & $1.03 \pm 0.18$ \\
\hline \multirow{3}{*}{ OHI(S) } & Baseline & 0 & 0 & 0 \\
& Day14 & $0.25 \pm 0.09$ & $0.20 \pm 0.12$ & $0.73 \pm 0.26$ \\
& Day28 & $0.48 \pm 0.28$ & $0.32 \pm 0.18$ & $1.17 \pm 0.37$ \\
\hline
\end{tabular}

‡ PI- Plaque index; GI - Gingival index; OHI(S) - Oral hygiene index simplified; SD - Standard deviation

Intergroup comparisons of the clinical parameters are summarized in Table 2. A significant difference in PI, $\mathrm{GI}$, and $\mathrm{OHI}(\mathrm{S})$ was found when group 1 and group II was compared with group 3 on day14 and day 28 .

Table 2. Consolidated Pairwise Comparison (p-value) Among the Three Groups $(\mathrm{p}<0.05)$

\begin{tabular}{|l|l|l|l|}
\hline \hline Variable & Mean difference (95\% CI) & t & p \\
\hline PI & & & \\
Day 14 & & & \\
Group1 vs Group2 & $0.020(0.039-0.0008)$ & 1.054 & 0.2985 \\
Group1 vs Group3 & $-0.770(-0.89-0.64)$ & -12.676 & $<0.0001^{*}$ \\
Group2 vs Group3 & $-0.790(-0.91-0.66)$ & -13.005 & $<0.0001^{*}$ \\
Day28 & & & \\
\hline
\end{tabular}




\begin{tabular}{|l|l|l|l|}
\hline Group1 vs Group2 & $0.170(0.28--0.05)$ & 2.946 & $0.0055^{*}$ \\
Group1 vs Group3 & $-0.740(-0.91-0.56)$ & -8.646 & $<0.0001^{*}$ \\
Group2 vs Group3 & $-0.910(-1.07-0.75)$ & -11.515 & $<0.0001^{*}$ \\
\hline GI & & & \\
Day 14 & $0.010(0.03-0.01)$ & 0.894 & 0.3767 \\
Group1 vs Group2 & $-0.90(-1.06--0.73)$ & -10.815 & $<0.0001^{*}$ \\
Group1 vs Group3 & $-0.910(-1.07-0.75)$ & -10.963 & $<0.0001^{*}$ \\
Group2 vs Group3 & $0.160(0.25-0.06)$ & 3.392 & $0.0016^{*}$ \\
Day28 & $-0.580(-0.69--0.46)$ & -10.190 & $<0.0001^{*}$ \\
Group1 vs Group2 & $-0.740(-0.83-0.64)$ & -15.688 & $<0.0001^{*}$ \\
Group1 vs Group3 & & & \\
Group2 vs Group3 & $0.050(0.11-0.01)$ & 1.491 & 0.1443 \\
\hline OHI(S) & $-0.480(-0.60-0.35)$ & -7.802 & $<0.0001^{*}$ \\
Day 14 & $-0.530(-0.65-0.40)$ & -8.277 & $<0.0001^{*}$ \\
Group1 vs Group2 & $0.160(0.31--0.01)$ & 2.150 & $0.0380^{*}$ \\
Group1 vs Group3 & $-0.690(-0.90-0.48)$ & -6.650 & $<0.0001^{*}$ \\
Group2 vs Group3 & $-0.850(-1.03-0.66)$ & -9.239 & $<0.0001^{*}$ \\
Day28 & & & \\
Group1 vs Group2 & & & \\
Group1 vs Group3 & & & \\
Group2 vs Group3 & & & \\
\hline
\end{tabular}

*Statistically significant at $P<0.05$; $\mathrm{p}$ - Probability; Independent sample $t$-test; CI - Confidence interval, PI Plaque index; GI - Gingival index; OHI(S) - Oral hygiene index- simplified

\section{Discussion}

Chemical agents have been increasingly used as an adjunct to mechanical control. The use of a mouthwash augments maintenance of oral health through its antiplaque and antibacterial chemical properties.[8]

Chlorhexidine gluconate is the most widely used mouthwash, as it is effective against various pathogenic microorganisms, fungi, yeasts, and viruses. Chlorhexidine is a cationic bisbiguanide having low toxicity and broad-spectrum antibacterial activity. When used as a mouthwash, it has a flushing action; its effect in the oral cavity is attributed to its lethal effects on the bacteria. Chlorhexidine blocks the acidic groups of salivary glycoprotein, adsorbs to the extracellular polysaccharides of bacteria, and reduces its ability to bind to tooth surfaces, and lastly by competing with calcium ion agglutinating factors in plaque.[9] Chlorhexidine has been reported to have a number of local side effects. These side effects are brown discoloration of the teeth and tongue, oral mucosal erosion, and taste perturbation which encouraged the search for an alternative antiplaque agent.

Probiotic mouthwash contains living microbes, which influence the health of the host when used in adequate numbers by utilizing natural beneficial bacteria commonly found in healthy mouths to provide a natural defense against those bacteria thought to be harmful to teeth and gums.[10] Probiotic strains contain bacterial strains which are not harmful, do not develop antibiotic resistance, and are nontoxic.[11]

The results obtained showed that there was a significant improvement in gingival bleeding, plaque accumulation, and oral hygiene after 14 days in all three groups. In this study, it was observed that there was a significant difference in the mean PI, GI and $\mathrm{OHI}(\mathrm{S})$ between the Control and Chlorhexidine group, and control and Probiotic mouth rinses groups after 14 days and 28 days. $(P<0.001)$. Probiotic and Chlorhexidine groups caused fewer plaque accumulations compared with the Control group. However, there were no significant differences in the mean PI, GI and OHI(S) between the Probiotic and Chlorhexidine groups on the 14th day of examination. The results of our study are in accordance with studies done by Vivekanand et al.,[12] Harini and Anegundi, ${ }^{[13]}$ and Noordin andKamin ${ }^{[14]}$ Purunaik et al. ${ }^{[15]}$ 


\section{Conclusion}

The Probiotic mouth rinse tested was effective in reducing plaque accumulation and gingival inflammation. Chlorhexidine is considered to be the most effective anti-plaque agent, but with certain limitations. Hence, search for an effective and safe alternative to CHX mouthwash has led to the emergence of probiotics which appears to be a boon for the treatment of diseases. Therefore, the Probiotic mouth rinse has a potential therapeutic value and further long-term study is recommended to determine its efficacy.

\section{References}

1. Sharma U, Jain RL, Pathak A. A clinical assessments of the effectiveness of mouthwashes in comparison to tooth brushing in children. J Indian Soc Pedod Prev Dent 2004;22:38-44.

2. Stamatova I, Meurman JH. Probiotics and periodontal disease.Periodontol 2000 2009;51:141-51.

3. Haukioja A. Probiotic lactobacilli and bifidobacteria in the mouth - In vitro studies on saliva-mediated functions and acid production. Med Odontol 2009.

4. Caglar E, Kargul B, Tanboga I. Bacteriotherapy and probiotics role on oral health. Oral Diseases. 2005;11: 131-7.

5. Beltrami M, Bickel M, Baehni PC. The effect of supragingival plaque control on the composition of the subgingival microflora in human periodontitis. J Clin Periodontol 1987;14:161- 4.

6. Bral M, Brownstein CN. Antimicrobial agents in the prevention and treatment of periodontal diseases. Dent Clin North Am 1988;32:217-41.

7. Williams RC, Paquette DW. Periodontal disease as a risk for systemic disease. In: Lindhe J, editor. Clinical Periodontology and Implant Dentistry. 5th ed. U.K: Blackwell Munksgaard; 2008. p. 475-95.

8. Dhawan R, Dhawan S. Role of probiotics on oral health: a randomized, double-blind, placebocontrolled study. J Interdiscip Dentistry 2013;3:71-8.

9. McBain AJ, Bartolo RG, Catrenich CE, Charbonneau D, Ledder RG, Gilbert P. Effects of a chlorhexidine gluconate-containing mouthwash on the vitality and antimicrobial susceptibility $\mathrm{f}$ in vitro oral bacterial ecosystems. Appl Environ Microbiol 2003;69:4770-6.

10. Meurman JH. Probiotics: Do they have a role in oral medicine and dentistry? Eur J Oral Sci 2005;113:188-96.

11. Thakkar PK, Imranulla M, Naveen Kumar PG, Prashant GM, Sakeenabi B, Sushanth VH. Effect of probiotic mouthrinse on dental plaque accumulation: a randomized controlled trial. Dent Med Res 2013;1:7-12.

12. Vivekananda MR, Vandana KL, Bhat KG. Effect of the probiotic Lactobacilli reuteri (Prodentis) in the management of periodontal disease: A preliminary randomized clinical trial. J Oral Microbiol 2010;2.[DOI: 10.3402/jom.v2i0.5344].

13. Harini PM, Anegundi RT. Efficacy of a probiotic and chlorhexidine mouth rinses A short-term clinical study. J Indian Soc Pedod Prev Dent 2010;28:179-82.

14. Noordin K, Kamin S. The effect of probiotic mouth rinse on plaque and gingival inflammation. Ann Dent Univ Malaya 2007;14:19-25.

15. Purunaik S, Thippeswamy HM, Chavan SS. To evaluate the effect of probiotic mouthrinse on plaque and gingivitis among 15-16-year-old school children of Mysore City, India-randomized controlled trial. Global J Med Res 2014;24:9-14.

How to cite this Article: Kumar Vivek, Amrita, Pratap Mahender, Comparison of The Effectiveness of Probiotic and Chlorhexidine Mouth Rinses on Plaque Accumulation and Gingival Inflammation.

Int. J. Drug Res. Dental Sci., 2019; 1(1):1-5.

Source of Support: Nil, Conflict of Interest: None declared. 